\title{
JOINT WEAK TYPE INTERPOLATION ON LORENTZ-KARAMATA SPACES
}

\author{
MICHAL BATHORY
}

Abstract. We present sharp interpolation theorems, including all limiting cases, for a class of quasilinear operators of joint weak type acting between Lorentz-Karamata spaces over $\sigma$-finite measure. This class contains many of the important integral operators. The optimality in the scale of Lorentz-Karamata spaces is also discussed. The proofs of our results rely on a characterization of Hardy-type inequalities restricted to monotone functions and with power-slowly varying weights. Some of the limiting cases of these inequalities have not been considered in the literature so far.

Mathematics subject classification (2010): 26D10, 46E30, 46B70, 47B38, 47G10. inequalities.

Keywords and phrases: Real interpolation, joint weak type operators, Lorentz-Karamata spaces, Hardy

\section{REFERENCES}

[1] C. Bennett And K. Rudnick, On Lorentz-Zygmund spaces, Dissertationes Math. (Rozprawy Mat.), 175 (1980), 1-67.

[2] C. Bennett And R. Sharpley, Interpolation of Operators, Academic Press, Inc., Boston, 1988.

[3] N. H. Bingham, C. M. Goldie, and J. L. Teugels, Regular Variation, Cambridge University Press, Cambridge, 1987.

[4] M. CARro And J. Soria, Weighted Lorentz spaces and the Hardy operator, J. Funct. Anal. 112, 2 (1993), 480-494.

[5] D. E. Edmunds, P. GuRKa, AND B. OpIC, Double exponential integrability of convolution operators in generalised Lorentz-Zygmund spaces, Indiana Univ. Math. J. 44, 1 (1995), 19-43.

[6] D. E. Edmunds, R. Kerman AND L. PICK, Optimal Sobolev imbeddings involving rearrangementinvariant quasinorms, J. Funct. Anal. 170, 2 (2000), 307-355

[7] W. D. EVANS, B. OPIC AND L. PICK, Interpolation of operators on scales of generalized LorentzZygmund spaces, Math. Nachr. 182 (1996), 127-181.

[8] W. D. Evans, B. OpIC AND L. PICK, Real interpolation with logarithmic functors, J. Inequal. Appl. 7, 2 (2002), 187-269

[9] A. Gogatishvili, J. S. Neves And B. Opic, Optimality of embeddings of Bessel-potential-type spaces into Lorentz-Karamata spaces, Proc. R. Soc. Edinb. 134A (2004), 1127-1147.

[10] A. GogatishVILI, B. OPIC AND W. TREBELS, Limiting reiteration for real interpolation with slowly varying functions, Math. Nachr. 278, 1-2 (2005), 86-107.

[11] A. Gogatishvili And V. D. Stepanov, Reduction theorems for operators on the cones of monotone functions, J. Math. Anal. Appl. 405, 1 (2013), 156-172.

[12] P. GURKA AND B. OPIC, Sharp embeddings of Besov-type spaces, J. Comput. Appl. Math. 208, 1 (2007), 235-269.

[13] L. V. Kantorovich And G. P. Akilov, Functional Analysis, 2nd ed., Pergamon Press, OxfordElmsford, N.Y., 1982.

[14] J. S. Neves, Lorentz-Karamata spaces, Bessel and Riesz potentials and embeddings, Dissertationes Math. (Rozprawy Mat.) 405 (2002), 1-46.

[15] B. OpIC And A. Kufner, Hardy-type inequalities, Pitman Research Notes in Math. Series 219, Longman Sci \& Tech., Harlow, 1990. 
[16] E. SAWYER, Boundedness of classical operators on classical Lorentz spaces, Studia Math. 96, 2 (1989), 145-158.

[17] G. Sinnamon, Hardy's inequality and monotonicity, Function Spaces, Differential Operators and Nonlinear Analysis (editors P. Drábek and J. Rákosník), Conference Proceedings, Milovy, Czech Republic, 2004.

[18] V. D. Stepanov, Weighted norm inequalities of Hardy type for a class of integral operators, J. London Math. Soc. (2), 50, 1 (1994), 105-120.

[19] V. D. Stepanov, The weighted Hardy's inequality for nonincreasing functions, Trans. Amer. Math. Soc., 338, 1 (1993), 173-186.

[20] A. Zygmund, Trigonometric Series, 3rd ed., vol. 1, Cambridge University Press, 2002. 